\title{
RESPOSTAS ESTRATÉGICAS DE PROGRAMAS E PROFESSORES PARANAENSES DE MESTRADO/DOUTORADO EM ADMINISTRAÇÃO À AVALIAÇÃO DA CAPES: CONFIGURANDO PROPOSIÇÕES INSTITUCIONAIS A PARTIR DE REDES DE COOPERAÇÃO ACADÊMICA ${ }^{1}$
}

\section{STRATEGICAL ANSWERS OF PROGRAMS AND PROFESSORS PARANAENSES OF MASTER/DOCTOR'S DEGREES IN ADMINISTRATION TO THE EVALUATION OF THE CAPES: CONFIGURING INSTITUCIONAL PROPOSALS FROM NETS OF ACADEMIC COOPERATION}

\author{
João Marcelo Crubellate ${ }^{2}$ \\ Luciano Rossoni ${ }^{3}$ \\ Cristiane Marques Mello ${ }^{4}$ \\ Juanita Bruneau Valenzuela ${ }^{5}$
}

\begin{abstract}
RESUMO: O principal objetivo deste artigo é apresentar proposições acerca da relação entre respostas estratégicas de Programas de Pós-Graduação stricto sensu do Estado do Paraná às pressões institucionais relativas à avaliação realizada pela CAPES e a imersão em rede de cooperação acadêmica por parte dos professores daqueles programas. Conjugando elementos conceituais da nova teoria institucional e de análise de rede sociais, elaboramos treze proposições para estudo das respostas estratégicas dos pesquisadores e dos programas paranaenses ao processo de avaliação da CAPES nos últimos 2 triênios (2001-2003 e 2004-2006). A análise da rede de cooperação acadêmica foi feita utilizando-se algoritmos e rotinas disponíveis no software UCINET 6. Os dados, secundários, vieram dos currículos disponibilizados pelo CNPq, na Base Lattes, e foram capturados em meados do mês de maio de 2007. As proposições derivadas deste estudo preliminar permitirão, em momento posterior, avançar no estudo empírico da relação entre respostas estratégicas e características estruturais e relacionais de redes de co-autoria e, mais amplamente, a relação entre organizações e seus ambientes sob uma perspectiva recursiva de análise institucional.
\end{abstract}

PALAVRAS-CHAVE: respostas estratégicas; co-autoria; redes sociais; institucionalização; CAPES.

\begin{abstract}
The main objective of this article is to present proposals concerning the relation between strategical answers of Programs of After-Graduation stricto sensu of the State of the Parana to relative the institucional pressures to the evaluation carried through for the CAPES and the immersion in net of academic cooperation on the part of the professors of those programs. Conjugating conceptual elements of the new institucional theory and social analysis of net, we elaborate thirteen proposals for study of the strategical answers of the researchers and the paranaenses programs to the process of evaluation of the CAPES in last the 2 triênios (2001-2003 and 2004-2006). The analysis of the net of academic cooperation was made using available algorithms and routines in software UCINET 6. The data, secondary, had come of the resumes disponibilizados for the CNPq, in the Lattes Base, and had been captured in middle of the month of May of 2007. The proposals derived from this preliminary study will allow, at posterior moment, to advance in the empirical study of the relation between structural and relationary strategical and characteristic answers of co-authorship nets and, more widely, the relation between organizations and its environments under a recursiva perspective of institucional analysis.
\end{abstract}

KEY WORD: strategical answers; co-authorship; social nets; institutionalization; CAPES

\footnotetext{
${ }^{1}$ Artigo publicado no EnEPQ - I Encontro de Ensino e Pesquisa em Administração e Contabilidade - Recife / PE - 21 a 23 de novembro de 2007.

Artigo Recebido em 21.08.2008. Revisado por pares em 09.10.2008. Recomendado em 16.10.2008 por Denise Del Pra Netto Machado (editora). Publicado em 15.12.2008.

Organização Responsável pelo periódico: Universidade regional de Blumenau - FURB - www.furb.br/rn

${ }^{2}$ Departamento de Administração Geral e Aplicada - Universidade Federal do Paraná jmcrubellate@terra.com.br

${ }^{3}$ Doutorando na Universidade Federal do Paraná - Professor na Universidade Positivo - $\underline{\text { rossoni@gmail.com }}$

${ }^{4}$ Mestre em Administração - Professora de ensino superior - mellcris@gmail.com

${ }^{5}$ Mestre em Administração - Professora de ensino fundamental e médio - juanita.bruneau@gmail.com
} 


\section{INTRODUÇÃO.}

Uma vertente frutífera da análise organizacional vem se configurando, nos últimos anos, pela incorporação de pressupostos de entendimento da realidade social como produto da interação humana. Por mais que isso já se ia superando em outras áreas do conhecimento, em especial na Sociologia, a teoria das organizações, até não muito tempo, pouco espaço dava a noções relacionais dentro e entre organizações. As noções de fronteira organizacional e seu conseqüente impacto para a discussão das relações entre organizações e ambiente organizacional eram, por exemplo, marcadas por objetivismo e dicotomias no tratamento dos conceitos, sob a expectativa de clara delimitação entre sistemas sociais e seus contextos ambientais. Com o advento de abordagens teóricas interpretativas no campo dos estudos organizacionais na década de 1980, essa circunstância sofreu alteração.

Um foco importante dessas abordagens interpretativistas na teoria administrativa contemporânea é a aproximação entre nova teoria institucional e estudos em estratégia. As características e possibilidades dessa aproximação vêm sendo descritas na literatura recente (OLIVER, 1991; 1997; INGRAM e SILVERMAN, 2002; MACHADO-DA-SILVA, 2003; CRUBELLATE, GRAVE e MENDES, 2004; SEIFERT e MACHADO-DA-SILVA, 2007; FLECK, 2007; VASCONCELOS, QUEIROZ e GOLDSZMIDT, 2007) que, em resumo, relaciona interesses de atores sociais, restrições e oportunidades institucionais e a estratégia organizacional (ou variáveis a ela pertinentes). O modo de explicação dessa inter-relação marca as diferentes formas pelas quais a nova teoria institucional vem sendo incorporada na discussão relativa à estratégia em organizações, com destaque para a vertente que explica o impacto do contexto institucional pela via da imersão social dos atores em redes sociais. A noção de rede social é especialmente rica por permitir operacionalizar (inclusive longitudinalmente) o inter-afetamento entre respostas estratégicas e ambiente institucional, ao descrever a transmissão de atitudes, informações e bens (DE NOOY, MRVAR e BATAGELJ, 2005).

Com base nessa possibilidade analítica, nosso principal objetivo neste artigo é elaborar proposições a respeito da relação entre imersão de programas de pós-graduação em Administração do Estado do Paraná em redes de cooperação acadêmica e respostas estratégicas desses programas aos critérios de avaliação da CAPES. Nos dois triênios aqui considerados, essa avaliação teve como critérios a proposta dos programas; corpo docente; atividade de pesquisa; atividade de formação; corpo discente; teses e dissertações; produção intelectual e, no segundo triênio, inserção social.

Ressaltamos aqui algumas delimitações importantes para o entendimento dos apontamentos feitos no artigo, que se referem a: a) imersão em redes de cooperação é vista, no estudo, a partir da cooperação dos professores e pesquisadores dos quatro programas sediados no Estado do Paraná. $\mathrm{O}$ foco analítico recai sobre a rede de cooperação como um todo, isto é, sobre características estruturais da rede, de modo a se evitar uma possível falácia ecológica derivada do foco em pesquisadores isolados; b) quanto à delimitação temporal, as redes foram configuradas utilizando-se dados dos dois últimos triênios de avaliação da CAPES, isto é, 2001 a 2003 e 2004 a 2006, conforme procedimentos e métodos descritos no tópico relativo à metodologia; c) quanto às respostas estratégicas, tratadas neste artigo apenas no plano de proposições de estudo norteadoras da continuidade dos esforços de pesquisa, elas se referem a ações tomadas ou pelo menos admitidas como tomadas pelos programas. De certo que em se tratando de programas de pós-graduação, há que se compreender que características estruturais dessas organizações (ou unidades, como se queira) - razoável grau de autonomia por parte de seus membros e das próprias unidades em relação às 
Universidades onde se localizam, a adhocracia como marca estrutural dos grupos de trabalho, e outros aspectos - sugerem que o foco em tais respostas de fato reflete não apenas a ação de coordenadores de programa mas de todo o grupo, ou pelo menos da maioria de seus participantes docentes.

Este artigo representa exemplo das possibilidades decorrentes do entendimento da estratégia organizacional como um fenômeno social. Apesar da brevidade e simplicidade de tratamento das questões implicadas, ele reforça a expectativa da perspectiva institucional em organizações quanto a que as questões pertinentes ao social sejam tratadas como sendo o que elas são, isto é, socialmente construídas.

\section{ESTRATÉGIA ORGANIZACIONAL, INSTITUIÇÕES E IMERSÃO EM REDES SOCIAIS.}

Para autores como INGRAM e CLAY (2000) e INGRAM e SILVERMAN (2002), a afirmação central da nova teoria institucional é que atores sociais perseguem seus interesses dentro de uma estrutura de restrições institucionais. Por mais que essa definição contemple, de fato, vários elementos conceituais importantes para a definição da teoria institucional noções de agência e interesse, de ambiente e da relação entre atores pessoais ou organizacionais e ambientes - ela ainda parece demasiadamente vinculada a uma distinção objetiva entre organização e ambiente organizacional e marcada, em algum grau, pela dicotomia entre comportamentos ou ações e instituições. Tal dicotomia é típica das versões extremadas de natureza humana sub e supersocializada, presentes na teoria econômica e social (GRANOVETTER, 1985).

A partir da crítica e das propostas formuladas por autores como GRANOVETTER (1985), DIMAGGIO e POWELL (1991), DIMAGGIO (1988), OLIVER (1991), dentre outros, a nova teoria institucional caminhou paulatinamente para o reconhecimento de que o papel das instituições, no que tange à ação, é tanto restritivo quanto constitutivo, certamente com uma ou outra preponderância, de acordo com o contexto espaciotemporal. Esse desenvolvimento da teoria se deu exatamente pela correção do que DACIN, GOODSTEIN e SCOTT (2002) chamaram de “... relativa desatenção ao papel de interesse e agência no delineamento das ações" (p. 47), o que teve como conseqüência a recuperação das discussões de variação e mudança pela perspectiva institucional e igualmente, a incorporação da possibilidade de análise institucional da estratégia organizacional. Alguns focos fundamentais nessa correção de caminho foram linguagem ou discurso, poder, agência, interpretação, todos eles permitindo reafirmar o papel ativo dos atores sociais na construção de significados a partir dos quais instituições são percebidas e interpretadas, formando deste modo o substrato das ações (DACIN et al., 2002; KOSTOVA e ROTH, 2002; MEYER e ROWAN, 2006; PECI, VIEIRA e CLEGG, 2006; SEIFERT e MACHADO-DA-SILVA, 2007; ZILBER, 2002; 2007; GARUD, HARDY e MAGUIRE, 2007).

Para BIDWELL (2006) a teoria institucional se desenvolveu, já em seus primórdios, com essa dupla perspectiva, que ele reconhece como decorrente da dupla força que origina as instituições e que condicionam o processo de institucionalização: poder e seleção por força de mecanismos sócio-adaptativos. Deste modo, institucionalização decorre do uso do poder via mecanismos políticos à disposição das elites, mas também decorre de processo de emergência, via adaptação ao ambiente. Em conseqüência, se a institucionalização é “... um processo dirigido por mecanismos políticos" (BIDWELL, 2006, p. 39), por ações intencionais de atores em posição de poder, deve-se também reconhecer que "... instituições 
criam poder" (BIDWELL, 2006, p. 38). Esse princípio circular ou recursivo de configuração das próprias instituições foi que, quando reconhecido no âmbito da teoria institucional, permitiu tratar adequadamente o papel de interesses, intenção e consciência, por um lado, e de ambiente institucional, de outro, na explicação das ações visando permanência ou mudança. Igualmente é essa lógica que permitiu à teoria organizacional institucionalista incorporar a discussão de estratégia. É interessante observar o que destacaram (sem exemplificar, o que limita um pouco o argumento) INGRAM e SILVERMAN (2002), no que tange ao papel constitutivo das instituições, quando afirmaram que "alguns estudos analisam mudanças nas regras que governam indústrias específicas para mostrar que o aumento nas restrições legais resulta no florescimento daquelas indústrias" (p. 11). Parece evidenciar-se nesses autores uma suposição especialmente importante tanto para a teoria das organizações quanto para a estratégia e que se refere à proposição de que se a intenção estratégica de atores sociais - Estados, organizações e pessoas - é um importante fator explicativo da concepção de certos arranjos institucionais, seus efeitos não podem ser reduzidos a esse único elemento explicativo, devendo-se considerar o fato de que mesmo instituições públicas e privadas centralizadas (nos termos de INGRAM e SILVERMAN, 2002) apenas emergem dessas intenções - isto é, não são totalmente explicadas pela intenção (também em MACHADO-DA-SILVA, FONSECA e CRUBELLATE, 2005 e GARUD et al., 2007).

O fato teórico importante em tudo isso é que a teoria institucional sustenta hoje que os atores sociais podem conservar capacidade estratégica em face das várias formas institucionais (INGRAM e SILVERMAN, 2002; DIMAGGIO, 1988; ROBERTS e GREENWOOD, 1997). Na nova teoria institucional esse entendimento passa pela adequada explicação de processo de interação entre atores e seus contextos ambientais, e uma via promissora para isso vem se desenvolvendo a partir da noção de imersão em redes sociais (por exemplo, GRANOVETTER, 1985). Redes sociais se referem ao relacionamento entre atores sociais (ROSSONI, HOCAYEN-DA-SILVA e FERREIRA JUNIOR, 2006; HANNEMAN e RIDDLE, 2005) e sua análise se volta para a descrição da estrutura desse relacionamento, e não para a descrição de atributos, como em outros modelos de análise. Deste modo, a preocupação essencial da análise de redes se volta ao estudo de como atores individuais estão "... imersos em uma estrutura e como a estrutura emerge de micro-relações entre suas partes individuais" (HANNEMAN e RIDDLE, 2005, p. 7; também TICHY, TUSHMAN e FOMBRUN, 1979). Ou seja, o princípio de redes sociais é, de fato, um princípio de recursividade, o mesmo para o qual caminha a nova teoria institucional e, por esta razão (dentre outras secundárias), a análise dessas redes é um método especialmente valioso para aquela teoria. Ao pôr foco sobre o caráter relacional das organizações (TICHY et al., 1979; BORGATTI e FOSTER, 2003) e em especial por sua capacidade de gerar entendimento sobre a interação entre aspectos micro e macro sociais implicados no comportamento organizacional (TICHY et al., 1979), a abordagem de redes permite analisar processos de adaptação estratégica de organizações a seus ambientes, nas quais se evidenciam tanto pressões ambientais quanto a agência de atores pessoais (EMIRBAYER e GOODWIN, 1994) e organizacionais no atendimento daquelas pressões ou na resistência a elas.

\section{MÉTODOS E PROCEDIMENTOS}

Este artigo reporta estudo comparativo de casos referentes aos quatro programas de pós-graduação stricto sensu em Administração existentes no Estado do Paraná nos triênios de avaliação da CAPES compreendidos pelos anos de 2001-2003 (3 programas) e 2004-2006 (4 
programas). Os dados secundários utilizados para o estudo e relativos à cooperação acadêmica entre professores - referente à parceria na publicação de artigos científicos em anais e periódicos, no período sob investigação - foram coletados do site do CNPq, especificamente da Base Lattes de currículos disponibilizadas por aquela agência federal ${ }^{1}$. Selecionaram-se as relações de co-autoria por essas serem as relações de maior relevância para a análise da imersão em campos científicos (ACEDO, BARROSO, CASANUEVA e GALÁN, 2006; ROSSONI e MACHADO-DA-SILVA, 2007). Os autores procederam à identificação do nome dos professores atuantes em cada um dos programas analisados, por meio das respectivas páginas virtuais disponibilizadas pelos próprios programas. Em seguida, e de posse da lista de docentes, fez-se consulta à Base Lattes e captura dos currículos dos professores, analisados posteriormente mediante registro de número de publicação no período, identificação de parcerias com respectivas IES/programas e, finalmente, tabulação em matriz do software de análise. $\mathrm{O}$ fato de os autores pertencerem, enquanto professor e alunas, de um dos programas envolvidos no estudo, auxiliou na complementação de informações necessárias para a análise dos dados (principalmente informações referentes a mudanças de programa por parte de alguns dos professores envolvidos no estudo, no período analisado, e também identificação de parcerias, nos casos em que os currículos mencionavam apenas sobrenome e iniciais dos nomes dos autores).

Os dados do estudo foram analisados mediante algoritmos e rotinas de análise de redes visando não apenas representar graficamente a rede de cooperação, mas também calcular graus de intensidade de cooperação dos programas, distâncias geodésicas entre os pesquisadores, coeficiente de agrupamento, centralidade de grau (Degree Centrality) e centralidade de intermediação (Betweenness Centrality) na rede relativa ao Estado (algoritmos descritos por HANNEMAN e RIDDLE, 2005). Para esses cálculos e representação das redes utilizou-se o software Ucinet 6 (BORGATTI, EVERETT e FREEMAN, 2002). Cabe mencionar que as três primeiras rotinas permitem verificar a provável difusão de informações e influência dentro das redes, e as duas últimas permitem identificar os atores sociais mais relevantes naquela rede de cooperação.

\section{REDE DE COOPERAÇÃO ACADÊMICO-DOCENTE: DADOS DESCRITIVOS, APROXIMAÇÕES TEÓRICAS E PROPOSIÇÕES DE ESTUDO.}

A história da pós-graduação stricto sensu em Administração no Estado do Paraná começa oficialmente no ano de 1992, com o início das atividades do Programa de PósGraduação em Administração da Universidade Federal do Paraná, em Curitiba. Durante toda a década de 1990, essa foi a única opção de formação pós-graduada na área, dentro do Estado. Já no ano 2000 surgem dois programas, um sediado na Pontifícia Universidade Católica do Paraná (PUC-PR) e outro, o consórcio interinstitucional da Universidade Estadual de Maringá e Universidade Estadual de Londrina, no interior do Estado. Finalmente, em 2005, surge o programa do Centro Universitário Positivo - UnicenP, também na capital do Estado. Salvo, portanto, o caso da UFPR, os três outros programas são relativamente novos, e esse é um fator importante para se ponderar quando se considera a interação acadêmica entre professores dos cursos mantidos por essas IES. Dados gerais relativos a essa interação, nos triênios de 2001-2003 e 2004-2006, são apresentados no quadro abaixo. 


\begin{tabular}{l|cc}
\hline & $N^{\boldsymbol{o}}$ Docentes & $\begin{array}{c}\text { Média de Cooperação (Co-autoria / } \\
\boldsymbol{N}^{\boldsymbol{o}} \text { docentes) }\end{array}$ \\
\hline 1. UFPR & $19^{*}(16)$ & 3,72 \\
2. PUC-PR & 18 & 3,56 \\
3. UEM/UEL & $15^{* *}(13)$ & 2 \\
4. UNICENP & $9^{*}$ & 2,89 \\
\hline
\end{tabular}

Quadro 1 - Dados descritivos da cooperação acadêmica nos Programas do Paraná - 2001 a 2006.

Fonte: Dados extraídos de currículos disponibilizados na Base Lattes/CNPq, aqui e no restante do artigo.

* Os Programas 1 e 4 compartilham 3 professores (permanentes no Programa 4 no segundo triênio).

** Dois professores do Programa 3 passaram a atuar no Programa 4 no segundo triênio (2004-2006).

Em parênteses o número de autores considerados para a análise dos relacionamentos das instituições.

Observamos que os dois programas mais antigos e de maior tamanho, UFPR e PUCPR, apresentaram maior média de cooperação no período, com 3,72 e 3,56 laços respectivamente. Já o UNICENP apresentou média de 2,89 laços por autor, superando o consórcio UEL/UEM, que obteve média de dois laços por autor. Mesmo a diferença de cooperação não sendo significativa $(\mathrm{f}=1,144, \mathrm{p}=0,340)$, aceitou-se que alguns programas apresentam autores com maior colaboração, pois a amostra é pequena, acarretando em um erro do tipo $\mathrm{II}^{2}$.

Na Figura 1 estão representados os laços de cooperação acadêmica dos professores dos quatro programas. Os números representam docentes dos programas que estabeleceram alguma parceria de cooperação acadêmica, nos período sob análise. Deste modo, note-se que não estão representados na rede todos os professores daqueles programas, uma vez que vários deles não publicaram textos em co-autoria, no período. Especificamente, dos 56 professores que atuavam nos programas nos dois triênios (alguns deles em mais de um programa), 13 (aproximadamente 23,2\%) não aparecem no desenho da rede. Isso não significa, por si, que esses foram professores academicamente improdutivos no período, mas apenas que não estabeleceram vínculos de co-autoria. O dado é relevante, entretanto, pelo objetivo deste artigo, que é discutir não produtividade acadêmica e sim a cooperação acadêmica nos programas. Os números dentro dos nós indicam a instituição em que o pesquisador está vinculado, sendo tais instituições expostas na legenda da Figura 1.

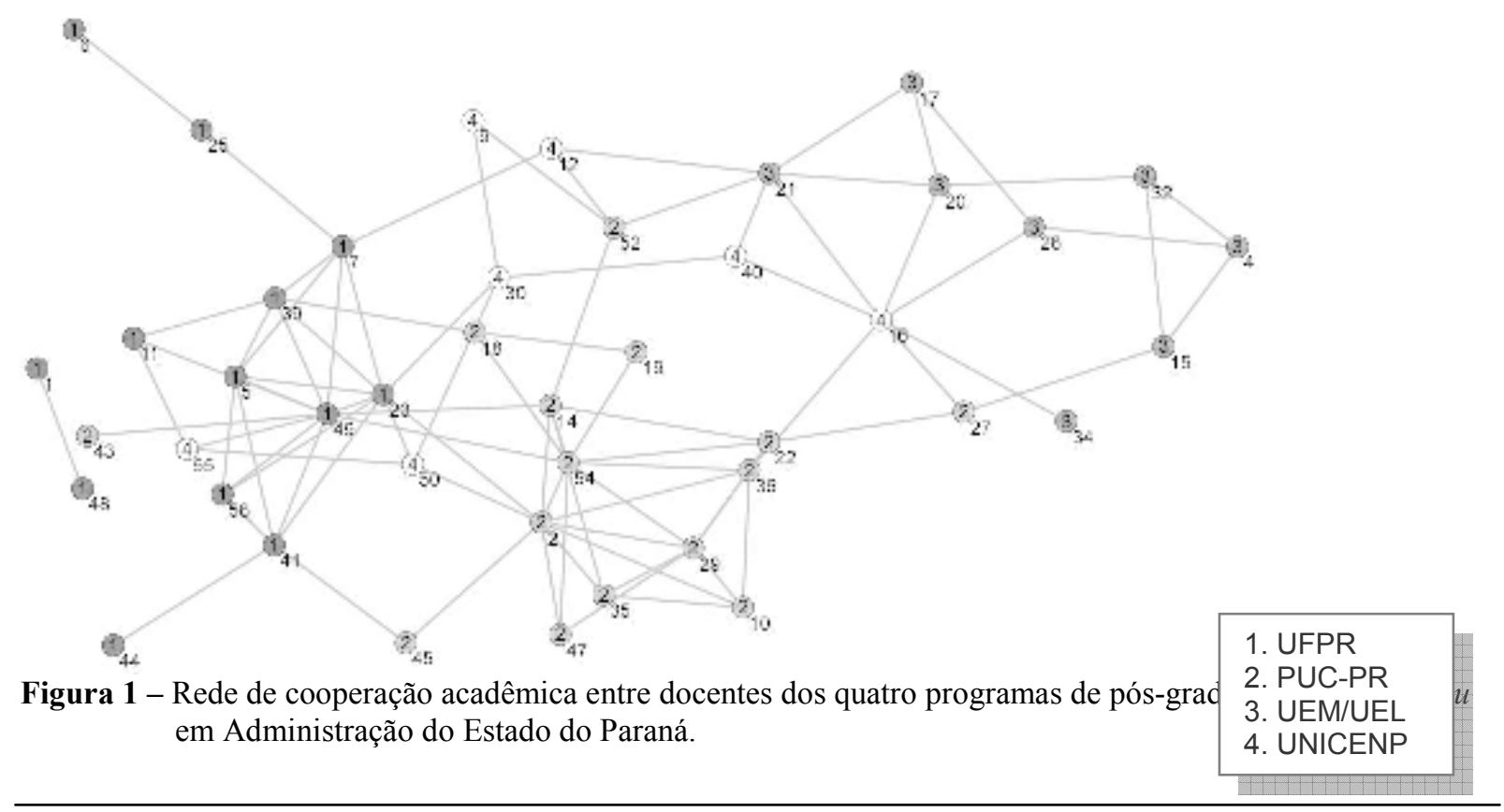

Revista de Negócios, ISSN 1980-4431, Blumenau, v13, n. 2, p. 77 - 92, Abril/Junho 2008. 
Uma análise visual da rede, conforme representada acima, permite observar que a cooperação para co-autoria dos professores do Programa 3 recebeu forte contribuição de professores que já não fazem parte do programa: os docentes representados pelos nós de número 40 e 16 são pontos de contato entre os Programas 3 e 4, sendo que tais cooperações ocorreram ainda no primeiro triênio, quando aqueles docentes ainda atuavam no Programa 3. Isso sinaliza para maior isolamento do Programa 3, no segundo triênio, visto que os pontos de contato passaram a se restringir a apenas dois docentes. De modo inverso, o Programa 4, apresenta baixa cooperação interna (entre professores do próprio programa) mas forte cooperação externa (com professores de outros programas), muito provavelmente em decorrência da presença de três docentes daquele programa também no Programa 1 e do pouco tempo de sua existência.

De acordo com ACEDO, BARROSO, CASANUEVA e GALÁN (2006), o grande motivador para a co-autoria em publicação científica é a expectativa do pesquisador em aumentar volume e qualidade de sua produção científica. Os mesmos autores, assim como BARABASI (2005), apontam também outros fatores, que poderíamos classificar como de ordem institucional e de ordem técnica, para explicar o crescente fenômeno da co-autoria (aspectos como aumento do grau de especialização no campo científico; divisão do trabalho intelectual pela expansão do estoque de conhecimento; crescimento do número de cientistas no campo; melhoria dos meios de comunicação; o padrão metodológico predominante nas disciplinas e seu grau de interdisciplinaridade). Da perspectiva mais ampla, portanto, os dados aqui analisados podem se relacionar - ou servir como indício - do grau em que os programas sob investigação são afetados ou do grau em que os professores atuantes nesses programas se definem como sendo afetados por amplas pressões institucionais, tanto quanto sugere modos de respostas a essas pressões, elaborados no âmbito daqueles programas. A análise institucional de respostas estratégicas sugere que os dois tipos de fatores acima mencionados se relacionam com as respostas estratégicas de organizações, em função de critérios de ganhos econômicos ou de dependência de recursos e de critérios de legitimidade (CASILE e DAVIS-BLAKE, 2002). Principalmente o critério de legitimidade na pósgraduação, tanto para programas, quanto para pesquisadores individuais é, atualmente, condicionado em elevado grau por quantidade e qualidade de publicação, constituindo-se então forte pressão ambiental em direção ao aumento da publicação por parte de professores e, como alternativa viável (conforme sugeriram ACEDO et al., 2006), aumento da cooperação para co-autoria. Secundariamente critérios vinculados a acesso a recursos também são importantes, posto que o acesso a bolsas de estudos para alunos, a recursos distribuídos por órgãos de fomento à pesquisa, a bolsas de pesquisa, no caso de pesquisadores individuais, dentre outros, são também dependentes - mesmo que indiretamente, em alguns casos - da contribuição de professores à produção acadêmica da área e da legitimidade de seus programas.

Para análise da configuração estrutural da rede de interação dos professores (e da interação em cada um dos programas), efetuamos cálculo de densidade, distância geodésica, coesão e coeficiente de agrupamento da rede geral dos programas (interprogramas) e da rede de cooperação de cada um dos programas, conforme Quadro 2: 


\begin{tabular}{l|ccccc}
\hline Programa & 1. UFPR & 2. PUC-PR & 3. UEM/UEL & 4. UNICENP & $\begin{array}{c}\text { Rede } \\
\text { Interprogramas }\end{array}$ \\
\hline Densidade & 0,1917 & 0,1765 & 0,1154 & 0,1389 & 0,0571 \\
Desvio Padrão & $(0,2667)$ & $(0,5621)$ & $(0,2308)$ & $(0,500)$ & $(0,1741)$ \\
$\begin{array}{l}\text { Distância Geodésica } \\
\text { Média }\end{array}$ & 0,3936 & 0,3812 & 0,3195 & 0,3458 & 0,2301 \\
$\begin{array}{l}\text { Coesão Baseada na } \\
\text { Distância (Compactness) }\end{array}$ & $0,6155)$ & $(1,6443)$ & $(0,7667)$ & $(2,0069)$ & $(1,1847)$ \\
$\begin{array}{l}\text { Coeficiente de } \\
\text { Agrupamento }\end{array}$ & 0,304 & 0,664 & 1,714 & 2,067 & 3,026 \\
\hline
\end{tabular}

Quadro 2 - Densidade, desvio padrão, distância geodésica e coesão das redes de cooperação dos Programas de Pós-Graduação em Administração do Paraná.

* Dados foram analisados como binários e simétricos.

** Dados em parênteses são simétricos, mas não são binários, pois levam em consideração a quantidade de artigos publicados em conjunto pelos autores.

A densidade de uma rede consiste na proporção de todos os laços possíveis na rede e que estão de fato presentes (HANNEMAN e RIDDLE, 2005; ACEDO et al., 2006; DE NOOY et al., 2005) e é medida importante para prever a velocidade de difusão de informações em uma rede e também o grau de capital social e de restrições sociais dos atores. Uma rede mais densa significa, em princípio, difusão mais rápida de informações. Avaliamos também a densidade considerando a freqüência da co-autoria, o que fornece indícios da intensidade da cooperação entre os pesquisadores dentro dos próprios programas. A distância geodésica e a coesão são medidas complementares à densidade, uma vez que redes mais densas apresentarão distâncias menores entre os atores (HANNEMAN e RIDDLE, 2005). Medidas de distância são importantes para prever tempo e intensidade de difusão de informações, de exercício de poder e influência entre atores imersos na rede, dentre outros aspectos. Distância geodésica implica "[...] no caminho mais curto possível entre um ator e outro" (HANNEMAN e RIDDLE, 2005, p. 87). Portanto, quanto menor o índice de uma rede, maior a probabilidade de fluxos intensos e rápidos e que alcancem todos na rede (daí ser ela uma medida de coesão). A compactabilidade é um subproduto do cálculo das distâncias geodésicas e o utilizamos aqui como complemento daquela. Os índices podem variar de 0 a 1; valores maiores significam maior coesão. Também utilizamos o coeficiente de agrupamento, que se baseia no agrupamento de atores em relação aos atores vizinhos, medindo a densidade e o grau de aninhamento entre esses autores (WATTS e STROGATTS, 1998). Quanto maior esse indicador, mais agrupada localmente a rede está, variando de 0 a 1 , do menos para o mais agrupado.

No caso dos dados relativos à cooperação entre pesquisadores dos Programas de Pósgraduação em Administração do Paraná, observa-se que a densidade é baixa $(0,0571)$. No entanto observa-se que a densidade entre pesquisadores pertencentes ao mesmo programa é bem superior ao da rede como um todo, havendo maior densidade entre os pesquisadores da UFPR $(0,1917)$ e menor densidade entre os pesquisadores do consórcio UEM/UEL $(0,1154)$. Diante da maior densidade entre pesquisadores entre programas, observa-se certo grau de homofilia nas relações, em que pesquisadores pertencentes a uma mesma categoria (nessa análise, pertencentes a uma mesma instituição) tendem a interagir mais entre si. Todavia, tais medidas de densidade não levam em consideração a intensidade e a freqüência dos relacionamentos entre os professores. Assim, avaliamos a densidade das relações considerando o número de vezes que os pesquisadores cooperaram entre si. Tais dados estão expostos entre parênteses no quadro 2. Observa-se que a instituição que apresentou maior 
densidade (UFPR), não apresentou grande diferenciação na densidade, quando considerada o número de co-autorias, o que nos leva a entender que nesse programa a cooperação é, na média, pouco freqüente e/ou bastante heterogênea. Também o consórcio UEM/UEL apresentou pequena diferença na densidade, quando considerado o número de autorias, mas, aparentemente, tais colaborações tendem a ser mais freqüentes do que a UFPR. Já o contrário ocorreu com os programas da PUC-PR e do UnicenP, que apresentaram grande densidade na cooperação, quando avaliado o número de co-autorias. Esses dados indicam que, mesmo não cooperando intensamente com a maior parte dos professores, as parcerias tendem a ser mais intensas e freqüentes do que na média da rede interprogramas como um todo. Mesmo havendo grandes diferenças em relação às duas medidas de densidade, consideraremos a densidade sem levar em conta a freqüência, pois tal medida é mais coerente para se avaliar as respostas dos programas (ou seja, mais importante, neste sentido, é o número de cooperação que a sua intensidade).

Já em relação à medida de distância, todos os programas apresentam distâncias similares, tendo o programa do consórcio UEM-UEL, menor distância do que os demais. Todavia, observando-se a compactabilidade, o consórcio UEM-UEL demonstrou-se mais fragmentado que os demais. Isso significa que naquele programa há barreiras à difusão de informações e exercício de influência dos atores entre si, no limite do que a co-autoria pode ser indício deste tipo de interação (neste artigo supomos, obviamente, que sim).

Por fim, avaliando-se o coeficiente de agrupamento dos programas, observa-se que UFPR e PUC-PR apresentam maior agrupamento local, o que indica que existe tendência nesses programas de porções de pesquisadores estarem mais aninhados que os demais. $\mathrm{O}$ coeficiente de agrupamento do Programa UEL/UEM foi baixo, mas nada comparado ao programa da UnicenP, que não apresentou nenhuma tríade, levando esse programa a apresenta valor nulo de alinhamento. Sendo o programa o mais recente dos demais, provavelmente ainda não houve tempo hábil para o fomento de grupos consolidados de pesquisa, o que é visível por meio da medida de coeficiente de agrupamento.

Se é plausível supor que a interação para co-autoria resulta exatamente no que está implicado na noção de densidade de uma rede, isto é, velocidade no fluxo de informações, influência, etc, maior ou menor densidade em uma rede de co-autoria podem significar, em termos de respostas estratégicas a pressões ambientais, maior ou menor velocidade de resposta e maior ou menor consenso na elaboração da resposta. Considerando-se ainda o que sugeriram HANNEMAN e RIDDLE (2005), isto é, que redes com poucas ou fracas conexões indicam grupos com baixa solidariedade, que respondem mais lentamente a estímulos (certamente, pelo baixo grau de coesão) e, inversamente, redes com muitas conexões são “... mais capazes de responder rápida e efetivamente" (p. 49), podemos supor que haverá diferença no ritmo de resposta estratégica naqueles programas. Parece plausível supor que:

Proposição 1: Os Programas de Pós-Graduação em Administração 1 e 2 respondem mais rapidamente que os Programas 3 e 4 às pressões e orientações relacionadas à avaliação da qualidade provenientes da CAPES.

Proposição 2: Os Programas 1 e 2 (mas especialmente o segundo) apresentarão respostas internamente mais coesas às pressões e orientações provenientes da CAPES do que os Programas 3 e 4.

Proposição 3: Os Programas 3 e 4 apresentarão menor coesão nas respostas às pressões e orientações da CAPES, relativamente aos demais programas do Estado, possivelmente desenvolvendo mais de uma resposta ou, pelo menos, respostas não coerentes entre si, ao longo do tempo. 
Densidade significa também possibilidade de influência, presença de maiores possibilidades de ação e, simultaneamente, de maiores restrições ao comportamento. Atores centrais (isto é, aqueles com maior número de laços) tendem a ser mais previsíveis em suas ações que atores periféricos (aqueles com menor número de laços), porque o maior capital social implicado no número de laços sociais facilita ações alternativas mas, ao mesmo tempo, resulta em maiores restrições em função de compromissos implicados nos próprios laços. No caso de atores periféricos, o menor número de compromissos resulta em menor grau de restrições, menor número de canais de influência sobre a rede e, ao mesmo tempo, maior número de contatos (ou laços) com outros atores fora da rede.

Tais ponderações, conjugadas com a teoria institucional e, dentro dela, a suposição de recursividade entre agência e estrutura, permitem supor que as ações de atores centrais tenderão a ser mais aquiescentes a padrões predominantes, provocando mudanças apenas incrementais, mas com forte impacto, principalmente ao longo do tempo. Já ações de atores periféricos tenderão a divergir do padrão predominante (intencionalmente ou não), mas terão menor impacto na rede como um todo.

Essas ponderações nos servem para analisar a rede geral dos quatro programas, conforme os dados apresentados anteriormente (também nos permitem elaborar a Proposição 7 , relativa à centralidade dos atores, apresentada adiante). Possibilitam-nos também derivar proposições acerca das respostas estratégicas dos atores individualmente, já que propomos analisar tanto o nível dos programas, quanto o nível dos pesquisadores. No Quadro 1 observamos que apenas o Programa 3 apresenta baixo grau de interação externa. Entretanto, a Figura 1 sugere (pelo menos em termos intuitivos) que a interação externa direta do Programa 2 está concentrada em poucos atores. De fato, dentre os quatro Programas, apenas 1 e 4 parecem de fato interagir externamente com intensidade. Sabemos, então, que o Programa 2 tem a maior densidade entre os programas do Estado, isto é, que há maior interação interna. Idéias, informações e influência circulam com maior velocidade e intensidade lá. Por outro lado, os Programas 3, principalmente, e também o Programa 2 (em menor grau) mantiveram menos laços de co-autoria com os outros programas do Estado. Isso sugere que sua resposta estratégica sofre menor impacto (normativo e cognitivo) dos outros programas, ou seja, é construída mais internamente. A discussão da interação externa entre os programas remete à idéia de multiplexidade, em que se avalia a proporção de trocas entre os programas (DEGENNE e FORSÉ, 1999), especificamente na elaboração de pesquisas científicas. Assim, parece plausível supor que:

Proposição 4: As respostas estratégicas dos Programas 1 e 4 tenderão a ser similares, pois existe maior multiplexidade (devendo-se recordar que no caso do Programa 4 é possível que não se encontre uma única resposta coesa no grupo).

Proposição 5: As respostas estratégicas dos Programas 2 e 3 tenderão a ser diferentes das respostas dos outros dois programas do Estado (devendo-se recordar que no caso do Programa 3 é possível que não se encontre uma única resposta coesa no grupo).

Resta discutir o possível conteúdo das respostas daqueles programas, dentro do escopo da discussão de respostas estratégicas no âmbito da nova teoria institucional, isto é, considerando-se um contínuo de aceitação ou resistência. Para isso, acreditamos ser necessário agregar informações relativas a atores individuais dentro das redes de cooperação dos Programas. Supomos que o tipo de resposta dos Programas no período analisado dependa principalmente de quais atores individuais eram centrais na rede dos programas do Estado e da relação desses atores sociais com a fonte de pressão e orientação aqui estudada, qual seja, a CAPES (sabidamente composta, no que tange aos critérios de avaliação dos Programas, por professores membros dos próprios programas avaliados). Supomos que caso um programa 
específico tenha, entre seus professores, algum membro de comitê avaliador da CAPES, esse membro será um ponto de relação entre a CAPES e o próprio programa. Daí, se esse mesmo professor for um dos atores centrais na rede, é plausível supor que ele ocupará posição privilegiada no sentido de difusão de idéias, informações e influência favorável às orientações emanadas da CAPES. Em decorrência, nesses casos, parece plausível supor maior aquiescência ou compromisso do Programa (e dos programas com os quais aquele ator mantém interação) com a CAPES, por ele representada na rede.

Para identificar atores sociais centrais na rede geral dos programas, utilizamos duas medidas: Centralidade de Grau (Degree Centrality) e Centralidade de Intermediação (Betweenness centrality), ambas seguindo a abordagem de Freeman (HANNEMAN e RIDDLE, 2005). A primeira refere-se à quantidade de laços que um ator social possui com outros, na rede, e se traduz em posição de vantagem pela influência, menor dependência, proeminência e prestígio daí derivados (HANNEMAN e RIDDLE, 2005). A segunda medida refere-se ao fato de atores sociais intermediarem relações entre outros atores, o que lhes confere menor dependência em relação a atores individuais e, em sentido inverso, maior poder pela disponibilidade de vários canais de influência (HANNEMAN e RIDDLE, 2005).

O coeficiente de variação para Centralidade de Grau (desvio padrão pela média vezes 100), nesta rede, é de 87,84, o que aponta para elevada heterogeneidade entre os atores no que concerne à sua proeminência e influência. $\mathrm{O}$ mesmo é sugerido pelo alto desvio padrão relativo à centralidade de intermediação dos atores da rede (conforme Tabela 3). Apresentamos na Tabela 3 apenas os 15 docentes indicados como mais centrais na rede, de acordo com os procedimentos adotados. Fazendo a análise comparativa entre os resultados dos dois índices, identificamos 10 docentes como os mais centrais, considerando-se sua presença entre os principais, nas duas colunas da Tabela: do Programa 1, quatro docentes, dos quais um é membro do comitê avaliador da CAPES para a área; do Programa 2, três docentes, dos quais um é representante do comitê avaliador da CAPES; do Programa 3, um docente; do Programa 4, dois docentes. Em face desses dados, nos pareceu pertinente propor que:

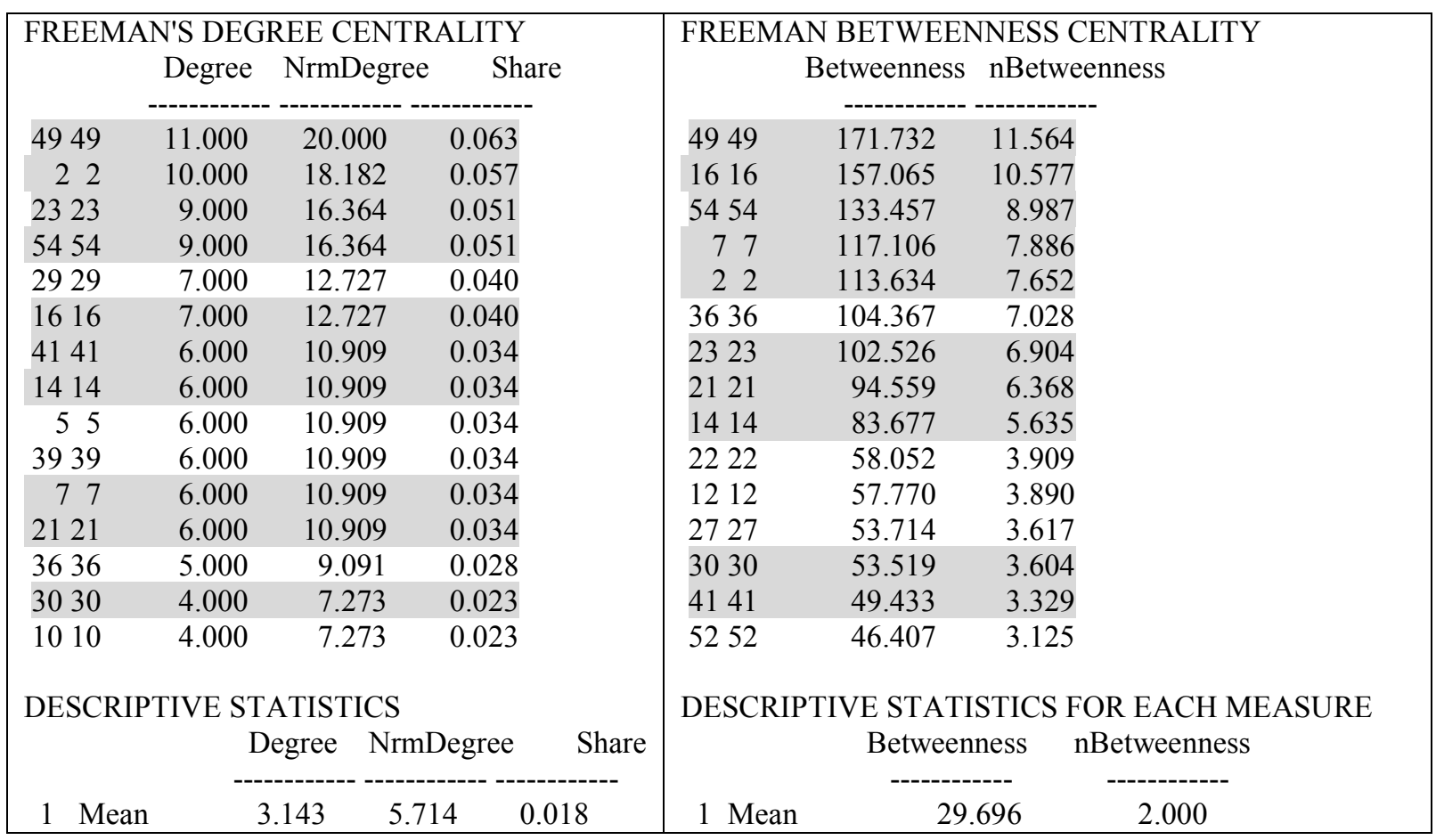

Revista de Negócios, ISSN 1980-4431, Blumenau, v13, n. 2, p. 77 - 92, Abril/Junho 2008. 


\begin{tabular}{|c|c|c|c|c|c|c|}
\hline 2 Std Dev & 2.761 & 5.020 & 0.016 & 2 Std Dev & 44.368 & 2.988 \\
\hline 3 Sum & 176.000 & 320.000 & 1.000 & 3 Sum & 1663.000 & 111.987 \\
\hline 4 Variance & 7.622 & 25.198 & 0.000 & 4 Variance & 1968.538 & 8.927 \\
\hline $5 \mathrm{SSQ}$ & 980.000 & 3239.669 & 0.032 & $5 \mathrm{SSQ}$ & 159623.281 & 723.841 \\
\hline 6 MCSSQ & 426.857 & 1411.098 & 0.014 & 6 MCSSQ & 110238.125 & 499.895 \\
\hline 7 Euc Norm & 31.305 & 56.918 & 0.178 & 7 Euc Norm & 399.529 & 26.904 \\
\hline 8 Minimum & 0.000 & 0.000 & 0.000 & 8 Minimum & 0.000 & 0.000 \\
\hline 9 Maximum & 11.000 & 20.000 & 0.063 & 9 Maximum & 171.732 & 11.564 \\
\hline \multicolumn{4}{|c|}{$\begin{array}{l}\text { Network Centralization }=14.81 \% \\
\text { Heterogeneity }=3.16 \% . \text { Normalized }=1.40 \%\end{array}$} & \multicolumn{3}{|c|}{ Network Centralization Index $=9.74 \%$} \\
\hline
\end{tabular}

Tabela 3 - Índices de Centralidade de Grau e de Centralidade de Intermediação dos 15

Docentes mais Centrais na Rede de Programas do Paraná.

Proposição 6: Os Programas 1 e 2 tenderão a aceitar em maior grau as pressões e orientações da CAPES (respostas do tipo aquiescência e compromisso) do que os Programas 3 (principalmente) e 4 (em menor grau).

De igual modo, a maior presença de atores centrais nos Programas 1, 2 e 4 sugere também que mudanças promovidas nesses programas, quando divergentes das orientações da CAPES, serão apenas incrementais, conforme discussão teórica que desenvolvemos anteriormente. Assim, parece-nos plausível supor que:

Proposição 7: As respostas estratégicas dos Programas 1, 2 e 4 às pressões e orientações da CAPES implicarão em mudanças apenas incrementais naqueles programas; as respostas do Programa 3 poderão provocar mudanças mais intensas.

Também analisamos os dados de co-autoria dos professores dos programas do Estado do Paraná com professores de outros programas localizados em outros Estados. A Figura 2, abaixo, indica os programas com os quais os professores do Paraná mantêm maior interação (em termos de quantidade absoluta de publicação conjunta). Os dados representados na Figura 2 sugerem que os professores paranaenses mantém maior interação de co-autoria com professores de três outros Programas: USP, FGV de São Paulo e Univali. Então, supomos que:

Proposição 8: As respostas estratégicas dos Programas do Paraná às pressões e orientações da CAPES tenderão a ser parecidas com as respostas estratégicas dos Programas da USP e da FGV, de São Paulo, e da Univali, de Santa Catarina.

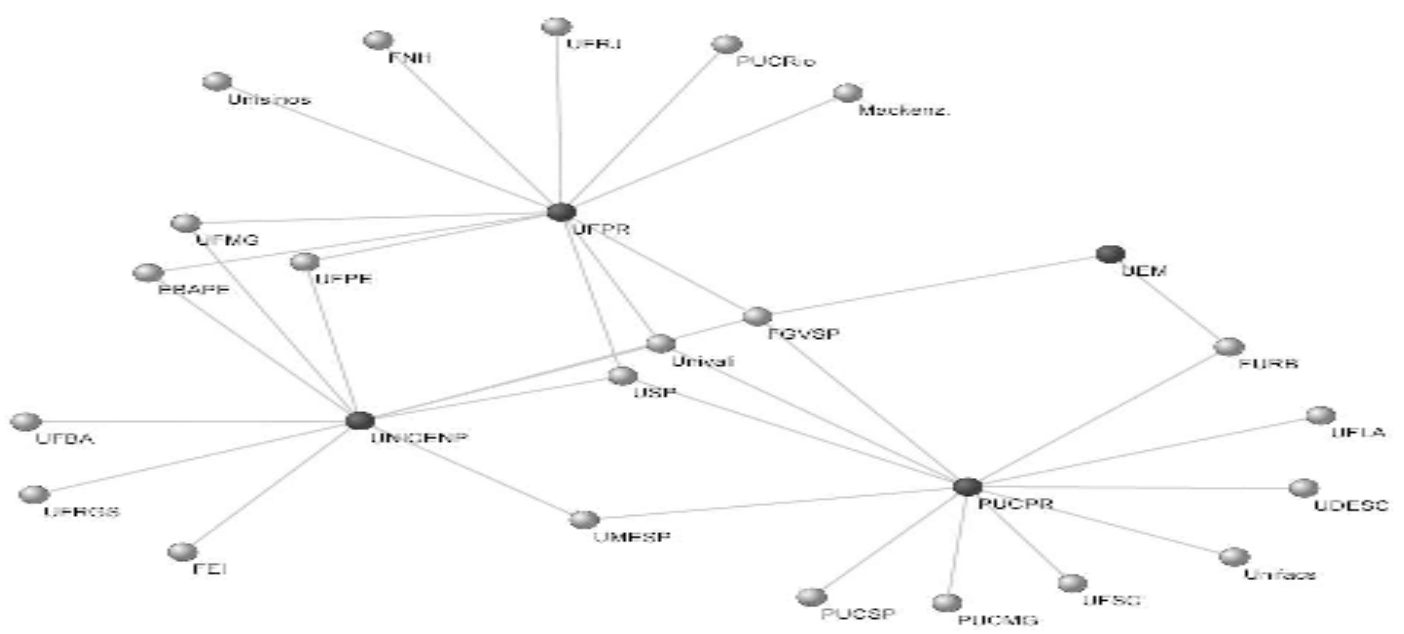

Figura 2 - Rede de Co-autoria entre Programas de Pós-Graduação em Administração do Paraná e Programas de Outros Estados. 
Por fim, diante da discussão sobre o papel individual dos pesquisadores, elaboramos um conjunto de proposições em relação ao nível individual. Mesmo que os programas apresentem certos padrões de respostas, sabemos que essas são adotadas ou rejeitadas de forma desigual pelos pesquisadores dos programas. A teoria institucional tem como premissa que entidades relacionais são condutoras de mecanismos normativos entre os pesquisadores (SCOTT, 2001). Esses mecanismos, diante da liberdade de conduta que os docentes apresentam (principalmente em instituições públicas) provavelmente condicionam as respostas dos pesquisadores em maior grau do que as regras estabelecidas pelos programas. Dessa forma, supomos que:

Proposição 9: A similaridade das respostas estratégicas dos pesquisadores é maior entre grupos estruturalmente similares do que entre autores dos mesmos programas.

Ainda no escopo individual de respostas, esperamos que o posicionamento dos pesquisadores em relação à sua produtividade, às suas díades e aos seus agrupamentos, tanto quanto mediante o destaque que este apresente em termos de centralidade da rede, condicione seus padrões de respostas, como discutido anteriormente. Portanto, parece plausível admitir que:

Proposição 10: Pesquisadores com maior centralidade de grau tendem a responder mais rapidamente (e com maior conformidade) às pressões da CAPES.

Proposição 11: Pesquisadores com maior número de artigos publicados tendem a responder mais rapidamente (e com maior conformidade) às pressões da CAPES.

Proposição 12: A resposta estratégica dos pesquisadores tende a ser similar às respostas de seus colaboradores diretos.

Ainda mediante às respostas individuais dos autores, deve-se observar outros mecanismos além dos normativos e relacionais na composição das respostas estratégicas dos professores. Alguns programas podem se utilizar de punições aos professores que não atenderem às delimitações relativas à conduta acadêmica, por exemplo, meta de artigos publicados, de pontuação da CAPES, número de orientações e de colaborações, entre outros. Dessa forma, espera-se que tais punições aumentem a conformidade em relação ao programa o que pode levar a maior isomorfismo nos padrões de respostas dentro do programa. Assim, esperamos que:

Proposição 13: Autores tendem a apresentar respostas similares dentro de um programa de pós-graduação quando esse apresenta mecanismos coercitivos de controle.

\section{CONCLUSÕES.}

As análises apresentadas neste artigo permitiram elaborar proposições para estudo da relação entre características da rede social de cooperação para co-autoria entre professores dos Programas de Pós-Graduação em Administração do Paraná e respostas estratégicas daqueles Programas a pressões e orientações da CAPES. A continuidade do estudo, com a captura e análise de dados relativos àquelas respostas estratégicas, permitirá analisar importantes relações teóricas que se encontram no centro das atenções de parte significativa dos teóricos das organizações, em especial das vertentes da teoria institucional e da análise de redes.

As análises aqui desenvolvidas estão sujeitas a algumas limitações. Dentre elas destacamos que as medidas de centralidade utilizadas indicam poder, influência, etc, mas são duas medidas dentre várias possíveis, na própria análise de redes e em outras vertentes teóricas. Além disso, sabe-se que a co-autoria pode indicar essa relação de poder, mas há 
outras formas de relação acadêmica e de outras naturezas que certamente interferem nesse poder e que não estão contempladas nas proposições deste artigo. Apesar destas limitações, acreditamos que o teste das proposições deste artigo servirá para evidenciar o quanto a coautoria de fato permite entender relações de poder em organizações de natureza acadêmicocientífica. Se essas proposições - ou pelo menos algumas delas - puderem ser corroboradas, então haverá grande possibilidade de utilização do contexto empírico da pós-graduação em Administração no Paraná (ou em contextos diferentes e mais amplos) como recurso para entendimento das relações de recursividade entre ações organizacionais e ambiente institucional, atendendo parte da agenda conjunta de pesquisa da nova teoria institucional e da estratégia em organizações nesta metade de década.

\footnotetext{
${ }^{1}$ Este se constitui em um limitante do estudo, uma vez que sabidamente a base Lattes ainda não é totalmente confiável no que tange às informações quanto a co-autoria. Entretanto, acreditamos que isso não invalide o estudo, principalmente pelo fato de que estamos analisando dados recentes.

${ }^{2}$ Para verificar se realmente havia indícios de ocorrência de um erro Tipo II, simulamos o teste ANOVA com a amostra multiplicada por três vezes, encontrando valor $f=3,607$ e $p=0,015$.
}

\section{REFERÊNCIAS}

ACEDO, F.; BARROSO, C.; CASANUEVA, C.; GALÁN, J. Co-authorship in management and organizational studies: an empirical and network analysis. Journal of Management Studies, 43 (5), p. 957-983, 2006.

BARABASI, A. Network Theory - The Emergence of the Creative Enterprise, Science, 308, April, p. 639-641, 2005.

BIDBWELL, C. Varieties of institutional theory: traditions and prospects for educational research. In H. Meyer \& B. Rowan (Eds), The new institutionalism in education (pp. 33-50). Albany: State University of New York Press, 2006.

BORGATTI, S. P.; EVERETT, M. G.; FREEMAN, L. C. UCINET for Windows: Software for Social Network Analysis. Boston: Harvard Analytic Technologies, 2002.

BORGATTI, S. P.; FOSTER, P.. The network paradigm in organizational research: a review and typology. Journal of Management, 29 (6), p. 991-1013, 2003.

CASILE, M.; DAVIS-BLAKE, A. When accreditation standards change: factors affecting differential responsiveness of public and private organizations. Academy of Management Journal, 45 (1), p. 180-195, 2002.

CRUBELlATE, J.; GRAVE, P.; MENDES, A. A questão institucional e suas implicações para o pensamento estratégico. Revista de Administração Contemporânea, 8 (Edição Especial), p. 37-60, 2004.

DACIN, M.; GOODSTEIN, J.; SCOTT, W.. Institutional theory and institutional change. Academy of Management Journal, 45 (1), p. 45-57, 2002.

DEGENNE, A.; FORSÉ, M.. Introducing Social Networks. London: Sage Publications, 1999. 
DIMAGGIO, P.. Interest and agency in institutional theory. In L. Zucker (Ed), Institutional patterns and organizations: culture and environments (pp. 3-21). Chicago: Bellinger, 1988.

EMIRBAYER, M.; GOODWIN, J. Network analysis, culture and the problem of agency. American Journal of Sociology, 99 (6), May, p. 1411-54, 1994.

FLECK, D.. Institutionalization and organizational long-term success. Brazilian Administration Review, 4 (2), p. 64-80, 2007.

GARUD, R.; HARDY, C.; MAGUIRE, S.. Institutional entrepreneurship as embedded agency: an introduction to the special issue. Organization Studies, 28 (7), p. 957-969, 2007.

GRANOVETTER, M.. Economic action and social structure: the problem of embeddedness. American Journal of Sociology, 91 (3), p. 481-510, 1985.

HANNEMAN, R.; RIDDLE, M. Introduction to social network methods. Riverside: University of Califórnia, 2005 (published in digital form at http://faculty.ucr.edu/ hanneman/), 2005.

INGRAM, P.; CLAY, K.. The choice-within-constraints new institutionalism and implications for sociology. Annual Review of Sociology, 26, p. 525-546, 2000.

INGRAM, P.; SILVERMAN, B.. Introduction. In: INGRAM, P.; SILVERMAN, B. The new institutionalism in strategic management, (pp. 1-30). Amsterdam: Elsevier, 2002.

KOSTOVA, T.; ROTH, K.. Adoption of an organizational practice by subsidiaries of multinational corporations: institutional and relational effects. Academy of Management Journal, 45 (1), p. 215-233, 2002.

MACHADO-DA-SILVA, C.. Respostas estratégicas da Administração e Contabilidade ao sistema de avaliação da CAPES, Organizações \& Sociedade, 10 (28), p. 63-77, 2003.

MACHADO-DA-SILVA, C.; FONSECA, V.; CRUBELLATE, J. Unlocking the institutionalization process: insights for an institutionalizing approach. Brazilian Administration Review, 2 (1), p. 1-20, 2005.

DE NOOY, W.; MRVAR, A.; BATAGELJ, V.. Exploratory social network analysis with Pajek. New York: Cambridge University Press, 2005.

OLIVER, C. Strategic responses to institutional processes. Academy of Management Review, $16(1)$, p. 145-179, 1991.

PECI, A.; VIEIRA, M.; CLEGG, S. A construção do 'real' e práticas discursivas: o poder nos processos de institucionaliz(ação). Revista de Administração Contemporânea, 10 (3), p. 51-71, 2006. 
QUEIROZ, M.; VASCONCELOS, F.; GOLDSZMIDT, R. Economic rents and legitimacy: incorporating elements of organizational analysis institutional theory to the field of business strategy. Brazilian Administration Review, 4 (1), p. 51-65, 2007.

ROSSONI, L.; HOCAYEN-DA-SILVA, J.; JUNIOR, I. Aspectos estruturais da cooperação entre pesquisadores no campo da Administração Pública e Gestão Social: análise das redes entre instituições no Brasil. Anais do EnAPG, Brasil, 2., 2006.

ROSSONI, L.; MACHADO-DA-SILVA, C.. Coesão Estrutural e Construção do Conhecimento Científico no Campo da Estratégia. Anais do 3Es, Brasil, 3., 2007.

SEIFERT, R.; MACHADO-DA-SILVA, C. Environment, resources and interpretation: influences in the internationalization strategies of the food industries in Brazil. Brazilian Administration Review, 4 (2), p. 40-63, 2007.

TICHY, N.; TUSHMAN, M.; FOMBRUN, C. Social network analysis for organizations. Academy of Management Review, 4, p. 507-519, 1979.

WATTS, D. J.; STROGATZ, S. H.. Collective Dynamics of "Small-World" Networks, Nature, 393, June, p. 440-442, 1998.

ZILBER, T. Institutionalization as an interplay between actions, meanings, and actors: the case of a rape crisis center in Israel. Academy of Management Journal, 45 (1), p. 234-254, 2002 .

ZILBERT, T. Stories and the discursive dynamics of institutional entrepreneurship: the case of Israeli High-tech after the bubble. Organization Studies, 28 (7), p. 1035-1054, 2007. 